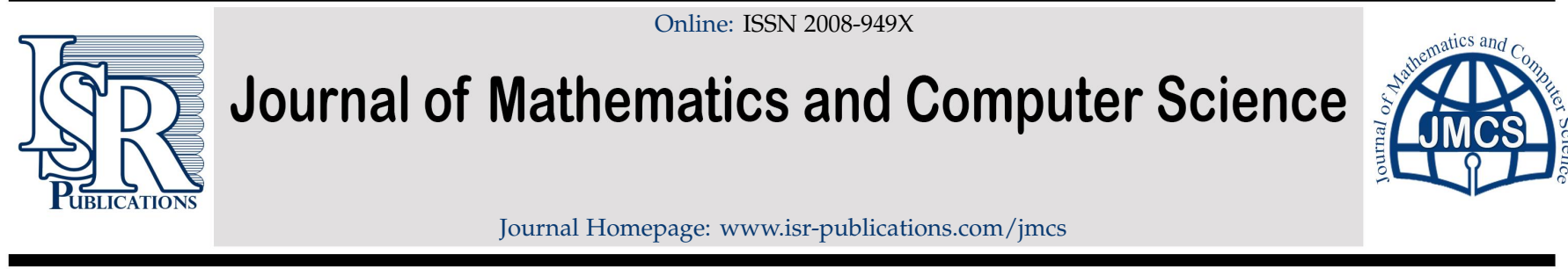

\title{
Maximal elements for Kakutani maps
}

\section{Donal O’Regan}

School of Mathematical and Statistical Sciences, National University of Ireland, Galway, Ireland.

\begin{abstract}
We present some new general existence theorems for maximal type elements for upper semicontinuous maps with convex compact values.
\end{abstract}

Keywords: Fixed and coincidence point theory, maximal elements.

2020 MSC: 47H10, 54H25.

(C)2022 All rights reserved.

\section{Introduction}

In this paper we present a variety of new collectively fixed point and coincidence point results and from these existence theorems we establish some new maximal type element results for majorized type maps (see [12]). The maps considered in this paper are Kakutani maps (or more generally, admissible maps with respect to Gorniewicz) and multivalued maps with continuous selections (see $[3,4,10,11]$ and the references therein).

Now we describe the maps considered in this paper. Let $\mathrm{H}$ be the C̆ech homology functor with compact carriers and coefficients in the field of rational numbers $\mathrm{K}$ from the category of Hausdorff topological spaces and continuous maps to the category of graded vector spaces and linear maps of degree zero. Thus $H(X)=\left\{H_{q}(X)\right\}$ (here $X$ is a Hausdorff topological space) is a graded vector space, $H_{q}(X)$ being the qdimensional Čech homology group with compact carriers of $X$. For a continuous map $f: X \rightarrow X, H(f)$ is the induced linear map $f_{\star}=\left\{f_{\star q}\right\}$ where $f_{\star q}: H_{q}(X) \rightarrow H_{q}(X)$. A space $X$ is acyclic if $X$ is nonempty, $\mathrm{H}_{\mathrm{q}}(X)=0$ for every $\mathrm{q} \geqslant 1$, and $\mathrm{H}_{0}(X) \approx \mathrm{K}$.

Let $X, Y$, and $\Gamma$ be Hausdorff topological spaces. A continuous single valued map $p: \Gamma \rightarrow X$ is called a Vietoris map (written $p: \Gamma \Rightarrow X$ ) if the following two conditions are satisfied:

(i) for each $x \in X$, the set $p^{-1}(x)$ is acyclic;

(ii) $p$ is a perfect map, i.e., $p$ is closed and for every $x \in X$ the set $p^{-1}(x)$ is nonempty and compact.

Let $\phi: X \rightarrow Y$ be a multivalued map (note for each $x \in X$ we assume $\phi(x)$ is a nonempty subset of $Y$ ). A pair $(p, q)$ of single valued continuous maps of the form $X \stackrel{p}{\leftarrow} \Gamma \stackrel{q}{\rightarrow} Y$ is called a selected pair of $\phi$ (written $(p, q) \subset \phi)$ if the following two conditions hold:

Email address: donal .oregan@nuigalway.ie (Donal O'Regan)

doi: $10.22436 /$ jmcs.027.01.07

Received: 2021-12-15 Revised: 2022-01-10 Accepted: 2022-01-20 
(i) $p$ is a Vietoris map; and

(ii) $\mathrm{q}\left(\mathrm{p}^{-1}(\mathrm{x})\right) \subset \phi(x)$ for any $x \in X$.

Now we define the admissible maps of Gorniewicz [7]. A upper semicontinuous map $\phi: X \rightarrow Y$ with compact values is said to be admissible (and we write $\phi \in \operatorname{Ad}(X, Y)$ ) provided there exists a selected pair $(p, q)$ of $\phi$. An example of an admissible map is a Kakutani map. A upper semicontinuous map $\phi: X \rightarrow K(Y)$ is said to Kakutani (and we write $\phi \in \operatorname{Kak}(X, Y)$ ); here $K(Y)$ denotes the family of nonempty, convex, compact subsets of $Y$.

The following classes of maps will play a major role in this paper. Let $Z$ and $W$ be subsets of Hausdorff topological vector spaces $Y_{1}$ and $Y_{2}$ and $G$ a multifunction. We say $G \in D K T(Z, W)$ [4] if $W$ is convex and there exists a map $S: Z \rightarrow W$ with $\operatorname{co}(S(x)) \subseteq G(x)$ for $x \in Z, S(x) \neq \emptyset$ for each $x \in Z$ and the fibre $S^{-1}(w)=\{z \in Z: w \in S(z)\}$ is open (in $Z$ ) for each $w \in W$. We say $G \in \operatorname{HLPY}(Z, W)[11]$ if $W$ is convex and there exists a map $S: Z \rightarrow W$ with $\operatorname{co}(S(x)) \subseteq G(x)$ for $x \in Z, S(x) \neq \emptyset$ for each $x \in Z$ and $Z=\bigcup\left\{\operatorname{int} S^{-1}(w): w \in W\right\}$.

Now we consider a general class of maps, namely the PK maps of Park. Let $X$ and $Y$ be Hausdorff topological spaces. Given a class $X$ of maps, $X(X, Y)$ denotes the set of maps $F: X \rightarrow 2^{Y}$ (nonempty subsets of $Y$ ) belonging to $X$, and $X_{c}$ the set of finite compositions of maps in $X$. We let

$$
\mathcal{F}(X)=\{Z: \text { Fix } F \neq \emptyset \text { for all } F \in X(Z, Z)\},
$$

where Fix F denotes the set of fixed points of $F$.

The class $\mathcal{U}$ of maps is defined by the following properties:

(i) $\mathcal{U}$ contains the class $\mathbf{C}$ of single valued continuous functions;

(ii) each $\mathrm{F} \in \mathcal{U}_{\mathrm{c}}$ is upper semicontinuous and compact valued; and

(iii) $B^{n} \in \mathcal{F}\left(\mathcal{U}_{c}\right)$ for all $n \in\{1,2, \ldots\}$; here $B^{n}=\left\{x \in \mathbf{R}^{n}:\|x\| \leqslant 1\right\}$.

We say $F \in P K(X, Y)$ if for any compact subset $K$ of $X$ there is a $G \in \mathcal{U}_{c}(K, Y)$ with $G(x) \subseteq F(x)$ for each $x \in K$. Recall PK is closed under compositions.

For a subset $K$ of a topological space $X$, we denote by $\operatorname{Cov}_{X}(K)$ the directed set of all coverings of $K$ by open sets of $X$ (usually we write $\operatorname{Cov}(\mathrm{K})=\operatorname{Cov}_{X}(\mathrm{~K})$ ). Given two maps $F, G: X \rightarrow 2^{Y}$ and $\alpha \in \operatorname{Cov}(\mathrm{Y}), \mathrm{F}$ and $G$ are said to be $\alpha$-close if for any $x \in X$ there exists $U_{x} \in \alpha, y \in F(x) \cap U_{x}$ and $w \in G(x) \cap U_{x}$.

Let $Q$ be a class of topological spaces. A space $Y$ is an extension space for $Q$ (written $Y \in E S(Q)$ ) if for any pair $(X, K)$ in $Q$ with $K \subseteq X$ closed, any continuous function $f_{0}: K \rightarrow Y$ extends to a continuous function $f: X \rightarrow Y$. A space $Y$ is an approximate extension space for $Q$ (written $Y \in A E S(Q)$ ) if for any $\alpha \in \operatorname{Cov}(Y)$ and any pair $(X, K)$ in $Q$ with $K \subseteq X$ closed, and any continuous function $f_{0}: K \rightarrow Y$ there exists a continuous function $f: X \rightarrow Y$ such that $\left.f\right|_{K}$ is $\alpha$-close to $f_{0}$.

Let $\mathrm{V}$ be a subset of a Hausdorff topological vector space $E$. Then we say $\mathrm{V}$ is Schauder admissible if for every compact subset $K$ of $V$ and every covering $\alpha \in \operatorname{Cov}_{V}(K)$ there exists a continuous function $\pi_{\alpha}: \mathrm{K} \rightarrow \mathrm{V}$ such that

(i) $\pi_{\alpha}$ and $i: \mathrm{K} \rightarrow \mathrm{V}$ are $\alpha$-close;

(ii) $\pi_{\alpha}(\mathrm{K})$ is contained in a subset $\mathrm{C} \subseteq \mathrm{V}$ with $\mathrm{C} \in \mathrm{AES}$ (compact).

$X$ is said to be q-Schauder admissible if any nonempty compact convex subset $\Omega$ of $X$ is Schauder admissible.

Theorem 1.1 ([1, 8]). Let $X$ be a Schauder admissible subset of a Hausdorff topological vector space and $\Psi \in$ $\mathrm{PK}(\mathrm{X}, \mathrm{X})$ a compact upper semicontinuous map with closed values. Then there exists an $\mathrm{x} \in \mathrm{X}$ with $\mathrm{x} \in \Psi(\mathrm{x})$.

Remark 1.2. Other variations of Theorem 1.1 can be found in [9].

We now list two well known results from the literature [12] (see also [2]). 
Theorem 1.3. Let $\mathrm{X}$ and $\mathrm{Y}$ be two topological spaces and $\mathrm{A}$ an open subset of $\mathrm{X}$. Suppose $\mathrm{F}_{1}: \mathrm{X} \rightarrow 2^{\mathrm{Y}}, \mathrm{F}_{2}: \mathrm{A} \rightarrow 2^{\mathrm{Y}}$ are upper semicontinuous such that $\mathrm{F}_{2}(\mathrm{x}) \subset \mathrm{F}_{1}(\mathrm{x})$ for all $\mathrm{x} \in \mathrm{A}$. Then the map $\mathrm{F}: \mathrm{X} \rightarrow 2^{\mathrm{Y}}$ defined by

$$
F(x)= \begin{cases}F_{1}(x), & x \notin A, \\ F_{2}(x), & x \in A\end{cases}
$$

is upper semicontinuous.

Theorem 1.4. Let $\mathrm{X}$ and $\mathrm{Y}$ be topological spaces. If $\mathrm{F}, \mathrm{G}: \mathrm{X} \rightarrow 2^{\mathrm{Y}}$ have compact values and are upper semicontinuous then $\mathrm{F} \cap \mathrm{G}$ is also upper semicontinuous.

We recall that a point $x \in X$ is a maximal element of a set valued map $F$ from a topological space $X$ to another topological space $Y$ if $F(x)=\emptyset$.

\section{Maximal element type results}

In this section we begin by presenting collectively fixed point results in a variety of settings. Our goal later is to consider collectively coincidence results.

Theorem 2.1. Let $\left\{X_{i}\right\}_{i=1}^{N}$ be a family of compact sets each in a Hausdorff topological vector space $E_{i}$. For each $i \in\{1, \ldots, N\}$ suppose $F_{i}: X \equiv \prod_{i=1}^{N} X_{i} \rightarrow X_{i}$ is upper semicontinuous with nonempty convex compact values (i.e., $\mathrm{F}_{\mathrm{i}} \in \operatorname{Kak}\left(\mathrm{X}, \mathrm{X}_{i}\right)$ ). Also assume $\mathrm{X}$ is a Schauder admissible subset of the Hausdorff topological vector space $E \equiv \prod_{i=1}^{N} E_{i}$. Then there exists an $x \in X$ with $x_{i} \in F_{i}(x)$ for $i \in\{1, \ldots, N\}$ (here $x_{i}$ is the projection of $x$ on $X_{i}$ ).

Proof. Let $F(x)=\prod_{i=1}^{N} F_{i}(x)$ for $x \in X$ and note $F \in \operatorname{Kak}(X, X)$ (see [2]). Now Theorem 1.1 guarantees the result.

\section{Remark 2.2.}

(i). Note we could replace $\left\{X_{i}\right\}_{i=1}^{N}$ with $\left\{X_{i}\right\}_{i \in I}$ (where I is an index set) in Theorem 1.1.

(ii). In Theorem 1.1 we could replace $F_{i} \in \operatorname{Kak}\left(X, X_{i}\right)$ with $F_{i} \in \operatorname{Ad}\left(X, X_{i}\right)$ (recall a finite product of admissible maps is admissible). This remark could also be applied to other results in this paper.

Theorem 2.3. Let $\left\{X_{i}\right\}_{i=1}^{N}$ be a family of sets each in a Hausdorff topological vector space $E_{i}$. For each $i \in\{1, \ldots, N\}$ let $\mathrm{K}_{i}$ be a nonempty compact subset of $\mathrm{X}_{i}$ and suppose $\mathrm{F}_{i}: \mathrm{X} \equiv \prod_{i=1}^{\mathrm{N}} \mathrm{X}_{i} \rightarrow \mathrm{K}_{i}$ is upper semicontinuous with nonempty convex compact values (i.e., $\mathrm{F}_{i} \in \operatorname{Kak}\left(\mathrm{X}, \mathrm{K}_{i}\right)$ ). Also assume $\mathrm{K} \equiv \prod_{i=1}^{\mathrm{N}} \mathrm{K}_{i}$ is a Schauder admissible subset of the Hausdorff topological vector space $\mathrm{E} \equiv \prod_{i=1}^{\mathrm{N}} \mathrm{E}_{i}$. Then there exists an $\mathrm{x} \in \mathrm{K}$ with $\mathrm{x}_{i} \in \mathrm{F}_{i}(\mathrm{x})$ for $i \in\{1, \ldots, N\}$.

Proof. Let $F(x)=\prod_{i=1}^{N} F_{i}(x)$ for $x \in K$ and note $F \in \operatorname{Kak}(K, K)$. Now apply Theorem 1.1.

Theorem 2.4. Let $\left\{X_{i}\right\}_{i=1}^{N}$ be a family of sets each in a Hausdorff topological vector space $E_{i}$. For each $i \in$ $\{1, \ldots, \mathrm{N}\}$ let $\mathrm{K}_{i}$ be a nonempty subset of $\mathrm{X}_{i}$ and suppose $\mathrm{F}_{i}: \mathrm{X} \equiv \prod_{i=1}^{\mathrm{N}} \mathrm{X}_{i} \rightarrow \mathrm{K}_{i}$. Let $\mathrm{F}: \mathrm{X} \rightarrow \mathrm{K}$ be given by $\mathrm{F}(\mathrm{X})=\prod_{i=1}^{\mathrm{N}} \mathrm{F}_{\mathrm{i}}(\mathrm{x})$ with $\mathrm{K} \equiv \prod_{i=1}^{\mathrm{N}} \mathrm{K}_{i}$ and assume $\mathrm{co}(\mathrm{K}) \subseteq \mathrm{X}$ is compact and $\mathrm{F}: \mathrm{X} \rightarrow \operatorname{co}(\mathrm{K})$ is upper semicontinuous with nonempty convex compact values (i.e., $\mathrm{F} \in \mathrm{Kak}(\mathrm{X}, \mathrm{co}(\mathrm{K}))$ ). Also suppose $\mathrm{co}(\mathrm{K})$ is a Schauder admissible subset of the Hausdorff topological vector space $\mathrm{E} \equiv \prod_{i=1}^{\mathrm{N}} \mathrm{E}_{i}$. Then there exists an $\mathrm{x} \in \operatorname{co}(\mathrm{K})$ with $x_{i} \in F_{i}(x)$ for $i \in\{1, \ldots, N\}$.

Proof. Note $\mathrm{F} \in \mathrm{Kak}(\operatorname{co}(\mathrm{K}), \operatorname{co}(\mathrm{K}))$ and apply Theorem 1.1.

The conclusion in the above results is the existence of a $x \in X$ with $x_{i} \in F_{i}(x)$ for $i \in\{1, \ldots, N\}$. One can adjust so that the conclusion is the existence of a $x \in X$ and a $i \in\{1, \ldots, N\}$ with $x_{i} \in F_{i}(x)$. To see this we will consider Theorem 2.1 in this setting. 
Theorem 2.5. Let $\left\{X_{i}\right\}_{i=1}^{N}$ be a family of convex compact sets each in a Hausdorff topological vector space $E_{i}$. For each $i \in\{1, \ldots, N\}$ suppose $F_{i}: X \equiv \prod_{i=1}^{N} X_{i} \rightarrow X_{i}$ is upper semicontinuous with convex compact values. Assume $\mathrm{X}$ is a Schauder admissible subset of the Hausdorff topological vector space $\mathrm{E} \equiv \prod_{i=1}^{\mathrm{N}} \mathrm{E}_{i}$. Also suppose for each $\mathrm{x} \in \mathrm{X}$ there exists an $\mathrm{i} \in\{1, \ldots, \mathrm{N}\}$ with $\mathrm{F}_{\mathrm{i}}(\mathrm{x}) \neq \emptyset$. Finally assume for each $\mathrm{i} \in\{1, \ldots, \mathrm{N}\}$ that $\mathrm{U}_{\mathrm{i}}=\left\{\mathrm{x} \in \mathrm{X}: \mathrm{F}_{\mathrm{i}}(\mathrm{x}) \neq \emptyset\right\}$ is open in $\mathrm{X}$. Then there exists an $\mathrm{x} \in \mathrm{X}$ and $a \mathrm{i}_{0} \in\{1, \ldots, \mathrm{N}\}$ with $\mathrm{x}_{\mathrm{i}_{0}} \in \mathrm{F}_{\mathrm{i}_{0}}(\mathrm{x})$.

Proof. Fix $i \in\{1, \ldots, N\}$. Define a map $G_{i}: X \rightarrow X_{i}$ by

$$
\left.G_{i}(x)\right)= \begin{cases}F_{i}(x), & x \in U_{i} \\ X_{i}, & x \in X \backslash U_{i}\end{cases}
$$

Note $G_{i}$ has nonempty convex compact values and $G_{i}: X \rightarrow X_{i}$ is upper semicontinuous from Theorem 1.3 (i.e., $G_{i} \in \operatorname{Kak}\left(X, X_{i}\right)$ ). Let $G: X \rightarrow X$ be given by

$$
G(x)=\prod_{j=1}^{N} G_{j}(x) \text { for } x \in X .
$$

Note $G \in \operatorname{Kak}(X, X)$. Now Theorem 1.1 guarantees a $y \in X$ with $y \in G(y))=\prod_{j=1}^{N} G_{j}(y)$, i.e., $y_{i} \in G_{i}(y)$ for $i \in\{1, \ldots, N\}$. Now by assumption there exists an $i_{0} \in\{1, \ldots, N\}$ with $F_{i_{0}}(y) \neq \emptyset$. Thus $y_{i_{0}} \in G_{i_{0}}(y)=$ $\mathrm{F}_{\mathrm{i}_{0}}(\mathrm{y})$.

Collectively fixed point theory can be rewritten as a maximal element type result. To illustrate this we will consider Theorems 2.1 and 2.5.

Theorem 2.6. Let $\left\{\mathrm{X}_{i}\right\}_{i=1}^{\mathrm{N}}$ be a family of convex compact sets each in a Hausdorff topological vector space $\mathrm{E}_{\mathfrak{i}}$. For each $i \in\{1, \ldots, N\}$ suppose $F_{i}: X \equiv \prod_{i=1}^{N} X_{i} \rightarrow X_{i}$ is upper semicontinuous with convex compact values. Also assume $X$ is a Schauder admissible subset of the Hausdorff topological vector space $E \equiv \prod_{i=1}^{N} E_{i}$. Now suppose for each $\mathrm{x} \in \mathrm{X}$ there exists $a \mathrm{j} \in\{1, \ldots, \mathrm{N}\}$ with $\mathrm{x}_{\mathrm{j}} \notin \mathrm{F}_{\mathrm{j}}(\mathrm{x})$. Then there exists an $\mathrm{x} \in \mathrm{X}$ and $a i_{0} \in\{1, \ldots, \mathrm{N}\}$ with $\mathrm{F}_{\mathrm{i}_{0}}(\mathrm{x})=\emptyset$.

Proof. Suppose the conclusion is false. Then for each $x \in X$ we have $F_{i}(x) \neq \emptyset$ for all $i \in\{1, \ldots, N\}$. Thus $F_{i} \in \operatorname{Kak}\left(X, X_{i}\right)$ for all $i \in\{1, \ldots, N\}$. Now Theorem 2.1 guarantees a $x \in X$ with $x_{i} \in F_{i}(x)$ for $i \in\{1, \ldots, N\}$, a contradiction.

Theorem 2.7. Let $\left\{X_{i}\right\}_{i=1}^{N}$ be a family of convex compact sets each in a Hausdorff topological vector space $E_{i}$. For each $i \in\{1, \ldots, N\}$ suppose $F_{i}: X \equiv \prod_{i=1}^{N} X_{i} \rightarrow X_{i}$ is upper semicontinuous with convex compact values. Assume $\mathrm{X}$ is a Schauder admissible subset of the Hausdorff topological vector space $\mathrm{E} \equiv \prod_{i=1}^{\mathrm{N}} \mathrm{E}_{i}$. Also suppose for all $i \in\{1, \ldots, N\}$ that $x_{i} \notin F_{i}(x)$ for each $x \in X$. Finally suppose for each $i \in\{1, \ldots, N\}$ that $U_{i}=\left\{x \in X: F_{i}(x) \neq \emptyset\right\}$ is open in $X$. Then there exists an $x \in X$ with $F_{i}(x)=\emptyset$ for all $i \in\{1, \ldots, N\}$.

Proof. Suppose the conclusion is false. Then for each $x \in X$ there exists an $i \in\{1, \ldots, N\}$ with $F_{i}(x) \neq \emptyset$. Now Theorem 2.5 guarantees an $x \in X$ and $a i \in\{1, \ldots, N\}$ with $x_{i} \in F_{i}(x)$, a contradiction.

We next discuss majorized type maps motivated from the literature (see [10, 12]). Let $Z$ and $W$ be sets in a Hausdorff topological vector space with $Z$ paracompact and $W$ convex and compact.

Remark 2.8. In the setting we presented above recall (i). compact sets are paracompact; and (ii). if $\Omega$ is a compact subset of a topological vector space then $\operatorname{co}(\Omega)$ is paracompact (see [4]).

Suppose $\mathrm{H}: \mathrm{Z} \rightarrow \mathrm{W}$ and for each $\mathrm{x} \in \mathrm{Z}$ assume there exists a map $A_{x}: Z \rightarrow W$ and an open set $\mathrm{U}_{x}$ containing $x$ with $\mathrm{H}(z) \subseteq A_{x}(z)$ for every $z \in U_{x}, A_{x}: U_{x} \rightarrow W$ is upper semicontinuous with convex compact values. We claim there exists a (compact) map $\Psi: Z \rightarrow W$ with $H(z) \subseteq \Psi(z)$ for $z \in Z$ and $\Psi: Z \rightarrow W$ is upper semicontinuous with convex compact values. To see this note $\left\{U_{x}\right\}_{x \in Z}$ is an open 
covering of $Z$ and since $Z$ is paracompact there exists $[5,6]$ a locally finite open covering $\left\{V_{x}\right\}_{x \in Z}$ of $Z$ with $x \in \mathrm{V}_{x}$ and $\mathrm{V}_{x} \subseteq \mathrm{U}_{\mathrm{x}}$ for $x \in \mathrm{Z}$, and for each $x \in \mathrm{Z}$ let

$$
\mathrm{Q}_{x}(z)= \begin{cases}\mathrm{A}_{\mathrm{x}}(z), & z \in \mathrm{V}_{\mathrm{x}} \\ W, & z \in \mathrm{Z} \backslash \mathrm{V}_{\mathrm{x}}\end{cases}
$$

Now Theorem 1.3 guarantees that $Q_{x}: Z \rightarrow W$ is upper semicontinuous with convex compact values. Next note $H(z) \subseteq Q_{x}(z)$ for every $z \in Z$ since if $z \in V_{x}$, then since $V_{x} \subseteq U_{x}$ and $H(w) \subseteq A_{x}(w)$ for $w \in \mathrm{U}_{x}$ we have $\mathrm{H}(z) \subseteq \mathrm{Q}_{x}(z)$ whereas if $z \in Z \backslash V_{x}$, then it is immediate since $\mathrm{Q}_{x}(z)=\mathrm{W}$. Now define $\Psi: Z \rightarrow W$ by

$$
\Psi(z)=\bigcap_{x \in Z} Q_{x}(z) \text { for } z \in Z .
$$

Note $\Psi: Z \rightarrow W$ has convex compact values with $\mathrm{H}(w) \subseteq \Psi(w)$ for $w \in Z$ since $\mathrm{H}(z) \subseteq \mathrm{Q}_{x}(z)$ for every $z \in Z$ (for each $x \in X$ ). It remains to show $\Psi: Z \rightarrow W$ is upper semicontinuous. Let $u \in Z$. There exists an open neighbourhood $N_{\mathfrak{u}}$ of $u$ such that $\left\{x \in Z: N_{\mathfrak{u}} \cap V_{x} \neq \emptyset\right\}=\left\{x_{1}, \ldots, x_{n_{u}}\right\}$ (a finite set). Note if $x \notin\left\{x_{1}, \ldots, x_{n_{u}}\right\}$, then $\emptyset=V_{x} \cap N_{\mathfrak{u}}$ so $Q_{x}(z)=W$ for $z \in N_{u}$ and so we have

$$
\Psi(z)=\bigcap_{x \in Z} Q_{x}(z)=\bigcap_{j=1}^{n_{u}} Q_{x_{j}}(z) \text { for } z \in N_{u} .
$$

Now for $j \in\left\{1, \ldots, n_{\mathfrak{u}}\right\}$ note $Q_{x_{j}}: Z \rightarrow W$ is upper semicontinuous (so $Q_{\chi_{j}}^{\star}: N_{\mathfrak{u}} \rightarrow W$, the restriction of $Q_{x_{j}}$ to $N_{u}$, is upper semicontinuous) so Theorem 1.4 guarantees that $\Psi: N_{u} \rightarrow W$ is upper semicontinuous (at $u$ ). Since $N_{u}$ is open we have that $\Psi: Z \rightarrow W$ is upper semicontinuous (at $u$ ).

We will combine the above discussion with Theorems 2.6 and 2.7 to illustrate the method involved.

Theorem 2.9. Let $\left\{X_{i}\right\}_{i=1}^{\mathrm{N}}$ be a family of convex compact sets each in a Hausdorff topological vector space $E_{i}$. For each $i \in\{1, \ldots, N\}$ suppose $H_{i}: X \equiv \prod_{i=1}^{N} X_{i} \rightarrow X_{i}$ and for each $x \in X$ assume there exists a map $A_{i, x}: X \rightarrow X_{i}$ and an open set $U_{i, x}$ containing $x$ with $\mathrm{H}_{i}(z) \subseteq A_{i, x}(z)$ for every $z \in U_{i, x}, A_{i, x}: U_{i, x} \rightarrow X_{i}$ is upper semicontinuous with convex compact values, and also assume for each $w \in X$ there exists a $j_{0} \in\{1, \ldots, N\}$ (which does not depend on $\mathrm{x}$ ) with $w_{j_{0}} \notin A_{j_{0}, x}(w)$. Suppose $X$ is a Schauder admissible subset of the Hausdorff topological vector space $\mathrm{E} \equiv \prod_{i=1}^{\mathrm{N}} \mathrm{E}_{\mathrm{i}}$. Then there exists an $\mathrm{x} \in \mathrm{X}$ and $a i_{0} \in\{1, \ldots, \mathrm{N}\}$ with $\mathrm{H}_{\mathrm{i}_{0}}(\mathrm{x})=\emptyset$.

Proof. Let $i \in\{1, \ldots, N\}$. From the discussion after Theorem 2.7 (with $Z=X, W=X_{i}, H=H_{i}$ and $\left.A_{x}=A_{i, x}\right)$ there exists a map $\Psi_{i}: X \rightarrow X_{i}$ with $H_{i}(z) \subseteq \Psi_{i}(z)$ for $z \in X$ and $\Psi_{i}: X \rightarrow X_{i}$ is upper semicontinuous with convex compact values: here $\left\{U_{i, x}\right\}_{x \in X}$ is an open covering of $X$ so there exists a locally finite open covering $\left\{V_{i, x}\right\}_{x \in X}$ of $X$ with $x \in V_{i, x}$ and $V_{i, x} \subseteq U_{i, x}$ for $x \in X$, and for each $x \in X$,

$$
\mathrm{Q}_{i, x}(z)= \begin{cases}\mathrm{A}_{i, x}(z), & z \in \mathrm{V}_{i, x} \\ X_{i,} & z \in X \backslash V_{i, x}\end{cases}
$$

and $\Psi_{i}: X \rightarrow X_{i}$ is

$$
\Psi_{i}(z)=\bigcap_{x \in X} Q_{i, x}(z) \text { for } z \in X .
$$

We now claim for each $w \in X$ there exists a $k \in\{1, \ldots, N\}$ with $w_{k} \notin \Psi_{k}(w)$. To see this fix $w \in X$. From our assumption for each $x \in X$ there exists a $j_{0} \in\{1, \ldots, N\}$ (which does not depend on $x$ ) with $w_{j_{0}} \notin A_{j_{0}, x}(w)$. Now since $\left\{V_{j_{0}, x}\right\}_{x \in X}$ is a covering of $X$ there exists an $x^{j_{0}} \in X$ with $w \in V_{j_{0}, x_{0} j_{0}}$ so

$$
\Psi_{j_{0}}(w)=\bigcap_{x \in X} Q_{j_{0}, x}(w) \subseteq Q_{j_{0}, x^{j_{0}}}(w)=A_{j_{0}, x^{j_{0}}}(w),
$$

and as a result $w_{j_{0}} \notin \Psi_{j_{0}}(w)$. Thus our claim is true. Now apply Theorem 2.6 (with $F_{i}=\Psi_{i}$ ) so there exists an $x \in X$ and $a i_{0} \in\{1, \ldots, N\}$ with $\Psi_{i_{0}}(x)=\emptyset$. Now since $H_{i}(z) \subseteq \Psi_{i}(z)$ for $z \in X$, then $H_{i_{0}}(x)=\emptyset$. 
Theorem 2.10. Let $\left\{X_{i}\right\}_{i=1}^{N}$ be a family of convex compact sets each in a Hausdorff topological vector space $E_{i}$. For each $i \in\{1, \ldots, N\}$ suppose $H_{i}: X \equiv \prod_{i=1}^{N} X_{i} \rightarrow X_{i}$ and for each $x \in X$ assume there exists a map $A_{i, x}: X \rightarrow X_{i}$ and an open set $U_{i, x}$ containing $x$ with $\mathrm{H}_{i}(z) \subseteq A_{i, x}(z)$ for every $z \in U_{i, x}, A_{i, x}: U_{i, x} \rightarrow X_{i}$ is upper semicontinuous with convex compact values, and also assume $w_{i} \notin A_{i, x}(w)$ for each $w \in X$. Suppose $X$ is a Schauder admissible subset of the Hausdorff topological vector space $E \equiv \prod_{i=1}^{N} E_{i}$. Finally assume for each $i \in\{1, \ldots, N\}$ that $\mathrm{U}_{i}=\left\{x \in X: H_{i}(x) \neq \emptyset\right\}$ is open in $X$. Then there exists an $x \in X$ with $H_{i}(x)=\emptyset$ for all $i \in\{1, \ldots, N\}$.

Proof. Let $i \in\{1, \ldots, N\}$ and let $V_{i, x}, Q_{i, x}$ and $\Psi_{i}$ be as in Theorem 2.9. We now claim that $w_{i} \notin \Psi_{i}(w)$ for each $w \in X$. To see this fix $w \in X$. Then there exists an $x^{\star} \in X$ with $w \in V_{i, x^{\star}}$ (recall $\left\{V_{i, x}\right\}_{x \in X}$ is a covering of $X)$ so

$$
\Psi_{i}(w)=\bigcap_{x \in X} Q_{i, x}(w) \subseteq Q_{i, x^{\star}}(w)=A_{i, x^{\star}}(w),
$$

and thus since $w_{i} \notin A_{i, x^{\star}}(w)$ we have $w_{i} \notin \Psi_{i}(w)$, and our claim is true.

Define a map $G_{i}: X \rightarrow X_{i}$ by

$$
G_{i}(x)= \begin{cases}\Psi_{i}(x), & x \in U_{i}=\left\{x \in X: H_{i}(x) \neq \emptyset\right\} \\ X_{i}, & x \in X \backslash U_{i}\end{cases}
$$

Note $G_{i}$ has nonempty convex compact values (to see the nonemptyness let $x \in X$ and note if $x \in X \backslash U_{i}$ then it is immediate whereas if $x \in U_{i}$, then $H_{i}(x) \neq \emptyset$ implies $\Psi_{i}(x) \neq \emptyset$ since $H_{i}(z) \subseteq \Psi_{i}(z)$ for $\left.z \in X\right)$ and $G_{i}: X \rightarrow X_{i}$ is upper semicontinuous from Theorem 1.3 (i.e., $G_{i} \in \operatorname{Kak}\left(X, X_{i}\right)$ ). Let $G: X \rightarrow X$ be given by

$$
G(x)=\prod_{j=1}^{N} G_{j}(x) \text { for } x \in X .
$$

Note $G \in \operatorname{Kak}(X, X)$. Now Theorem 1.1 guarantees a $y \in X$ with $y \in G(y))=\prod_{j=1}^{N} G_{j}(y)$, i.e., $y_{i} \in G_{i}(y)$ for $i \in\{1, \ldots, N\}$. If there exists an $i_{0} \in\{1, \ldots, N\}$ with $H_{i_{0}}(y) \neq \emptyset$ then $y \in U_{i_{0}}$ so $y_{i_{0}} \in G_{i_{0}}(y)=\Psi_{i_{0}}(y)$, a contradiction. Thus $H_{i}(y)=\emptyset$ for all $i \in\{1, \ldots, N\}$.

Now we present collectively coincidence results in a variety of settings.

Theorem 2.11. Let $\left\{X_{i}\right\}_{i=1}^{N},\left\{Y_{i}\right\}_{i=1}^{N_{0}}$ be families of convex sets each in a Hausdorff topological vector space $E_{i}$ and $\left\{Y_{i}\right\}_{i=1}^{N_{0}}$ is also a family of compact sets. For each $i \in\left\{1, \ldots, N_{0}\right\}$ suppose $F_{i}: X \equiv \prod_{i=1}^{N} X_{i} \rightarrow Y_{i}$ and $F_{i} \in \operatorname{Kak}\left(X, Y_{i}\right)$. For each $j \in\{1, \ldots, N\}$ suppose $G_{j}: Y \equiv \prod_{i=1}^{N_{0}} Y_{i} \rightarrow X_{j}$ and $G_{j} \in \operatorname{DKT}\left(Y, X_{j}\right)$. Then there exists an $\mathrm{x} \in \mathrm{X}$ and $a \mathrm{y} \in \mathrm{Y}$ with $\mathrm{y}_{\mathrm{j}} \in \mathrm{F}_{\mathrm{j}}(\mathrm{x})$ for $\mathrm{j} \in\left\{1, \ldots, \mathrm{N}_{0}\right\}$ and $\mathrm{x}_{\mathrm{i}} \in \mathrm{G}_{\mathrm{i}}(\mathrm{y})$ for $\mathrm{i} \in\{1, \ldots, \mathrm{N}\}$.

Proof. Now $Y$ is compact, $G_{i} \in \operatorname{DKT}\left(Y, X_{j}\right)$ so for each $i \in\{1, \ldots, N\}$ from $[3,4]$ there exists a continuous (single valued) selection $q_{i}: Y \rightarrow X_{i}$ of $G_{i}$ with $q_{i}(y) \in G_{i}(y)$ for $y \in Y$ and there exists a finite set $R_{i}$ of $X_{i}$ with $\mathrm{q}_{i}(Y) \subseteq \operatorname{co}\left(R_{i}\right) \equiv Q_{i}$. Let $Q=\prod_{i=1}^{N} Q_{i}(\subseteq X)$ and note $Q$ is compact. Let $F_{i}^{\star}$ denote the restriction of $F_{i}$ to $Q$ and let $F^{\star}(x)=\prod_{i=1}^{N_{0}} F_{i}^{\star}(x)$ for $x \in Q$. Note $F^{\star} \in \operatorname{Kak}(Q, Y)$ (so in particular $F^{\star} \in A d(Q, Y)$ ). Let $\mathrm{q}(\mathrm{y})=\prod_{i=1}^{N} q_{i}(y)$ for $y \in Y$ and note $q: Y \rightarrow Q$ is continuous (note $q_{i}: Y \rightarrow Q_{i}$ ). Then $q F^{\star} \in \operatorname{Ad}(Q, Q)$ (recall Ad maps are closed under compositions) and note $\mathrm{Q}$ is a compact convex in a finite dimensional subspace of $E=\prod_{i=1}^{N} E_{i}$, so Theorem 1.1 guarantees a $x \in Q$ with $x \in q\left(F^{\star}(x)\right)$. Now let $y \in F^{\star}(x)$ with $x=q(y)$. Note $y \in F(x)$ so $y_{j} \in F_{j}(x)$ for all $j \in\left\{1, \ldots, N_{0}\right\}$. Also $x_{i}=q_{i}(x) \in G_{i}(y)$ for $i \in\{1, \ldots, N\}$.

\section{Remark 2.12}

(i). In Theorem 2.11 we could replace $G_{j} \in \operatorname{DKT}\left(Y, X_{j}\right)$ with $G_{j} \in \operatorname{HLPY}\left(Y, X_{j}\right)$.

(ii). In Theorem 2.11 we could replace $\left\{X_{i}\right\}_{i=1}^{N},\left\{Y_{i}\right\}_{i=1}^{N_{0}}$ with $\left\{X_{i}\right\}_{i \in I},\left\{Y_{i}\right\}_{i \in J}$ (where I and J are index sets); here to apply Theorem 1.1 we need to assume $X$ is $q$-Schauder admissible. 
(iii). The assumption $\left\{Y_{i}\right\}_{i=1}^{N_{0}}$ are convex sets is not needed in the statement of Theorem 2.11.

(iv). In the proof of Theorem 2.11 note $F^{\star} q \in A d(Y, Y)$ so one could apply Theorem 1.1 if $Y$ is a Schauder admissible subset of $\prod_{i=1}^{N_{0}} E_{i}$. This remark could also be applied to other results in this section.

Theorem 2.13. Let $\left\{X_{i}\right\}_{i=1}^{N},\left\{Y_{i}\right\}_{i=1}^{N_{0}}$ be families of convex sets each in a Hausdorff topological vector space. For each $i \in\left\{1, \ldots, N_{0}\right\}$ suppose $F_{i}: X \equiv \prod_{i=1}^{N} X_{i} \rightarrow Y_{i}$ and $F_{i} \in \operatorname{Kak}\left(X, Y_{i}\right)$ and in addition assume there is a compact set $\mathrm{K}_{i}$ with $\mathrm{F}_{i}(\mathrm{X}) \subseteq \mathrm{K}_{i} \subseteq \mathrm{Y}_{i}$. For each $\mathrm{j} \in\{1, \ldots, \mathrm{N}\}$ suppose $\mathrm{G}_{j}: \mathrm{Y} \equiv \prod_{i=1}^{\mathrm{N}_{0}} \mathrm{Y}_{i} \rightarrow X_{j}$ and $\mathrm{G}_{j} \in \mathrm{DKT}\left(\mathrm{Y}, \mathrm{X}_{j}\right)$. Then there exists an $\mathrm{x} \in \mathrm{X}$ and $a \mathrm{y} \in \mathrm{Y}$ with $\mathrm{y}_{\mathrm{j}} \in \mathrm{F}_{\mathrm{j}}(\mathrm{x})$ for $\mathrm{j} \in\left\{1, \ldots, \mathrm{N}_{0}\right\}$ and $\mathrm{x}_{\mathrm{i}} \in \mathrm{G}_{\mathrm{i}}(\mathrm{y})$ for $\mathrm{i} \in\{1, \ldots, \mathrm{N}\}$.

Proof. Let $q_{i}, R_{i}, Q_{i}, Q, F_{i}^{\star}$ and $F^{\star}$ be as in Theorem 2.11. Note $F^{\star} \in \operatorname{Kak}(Q, Y)$ (so in particular $F^{\star} \in$ $\operatorname{Ad}(Q, Y))$. Now $F_{i}^{\star}(Q) \subseteq F_{i}(X) \subseteq K_{i}$ for each $i \in\left\{1, \ldots, N_{0}\right\}$ so $F^{\star}(Q) \subseteq K \equiv \prod_{i=1}^{N_{0}} K_{i}$. Let $q(y)=$ $\prod_{i=1}^{N} q_{i}(y)$ for $y \in Y$ and note $q F^{\star} \in A d(Q, Q)$ is a compact map. Also Theorem 1.1 guarantees a $x \in Q$ with $x \in \mathrm{q}\left(\mathrm{F}^{\star}(x)\right)$ and now the result follows from the argument in Theorem 2.11.

Theorem 2.14. Let $\left\{X_{i}\right\}_{i=1}^{N},\left\{Y_{i}\right\}_{i=1}^{N_{0}}$ be families of convex sets each in a Hausdorff topological vector space and $\left\{Y_{i}\right\}_{i=1}^{N_{0}}$ is also a family of compact sets. For each $i \in\left\{1, \ldots, N_{0}\right\}$ suppose $F_{i}: X \equiv \prod_{i=1}^{N} X_{i} \rightarrow Y_{i}$ is upper semicontinuous with convex compact values. For each $j \in\{1, \ldots, N\}$ suppose $G_{j}: Y \equiv \prod_{i=1}^{N_{0}} Y_{i} \rightarrow X_{j}$ and $\mathrm{G}_{j} \in \operatorname{DKT}\left(\mathrm{Y}, \mathrm{X}_{\mathrm{j}}\right)$. Also assume for each $\mathrm{x} \in \mathrm{X}$ there exists an $\mathrm{i} \in\left\{1, \ldots, \mathrm{N}_{0}\right\}$ with $\mathrm{F}_{i}(\mathrm{x}) \neq \emptyset$. Finally suppose for each $i \in\left\{1, \ldots, \mathrm{N}_{0}\right\}$ that $\mathrm{U}_{\mathrm{i}}=\left\{\mathrm{x} \in \mathrm{X}: \mathrm{F}_{\mathrm{i}}(\mathrm{x}) \neq \emptyset\right\}$ is open in $\mathrm{X}$. Then there exists an $\mathrm{x} \in \mathrm{X}, a \mathrm{y} \in \mathrm{Y}$ and $a$ $j_{0} \in\left\{1, \ldots, N_{0}\right\}$ with $y_{j_{0}} \in F_{j_{0}}(x)$ and $x_{i} \in G_{i}(y)$ for $i \in\{1, \ldots, N\}$.

Proof. Let $\mathrm{q}_{i}, \mathrm{R}_{i}, \mathrm{Q}_{i}$ and $\mathrm{Q}$ be as in Theorem 2.11. Let $i \in\left\{1, \ldots, \mathrm{N}_{0}\right\}$ and define a mapping $\Phi_{i}: \mathrm{X} \rightarrow \mathrm{Y}_{i}$ by

$$
\left.\Phi_{i}(x)\right)= \begin{cases}F_{i}(x), & x \in U_{i} \\ Y_{i}, & x \in X \backslash U_{i}\end{cases}
$$

Note $\Phi_{i}$ has nonempty convex compact values and $\Phi_{i}: X \rightarrow Y_{i}$ is upper semicontinuous from Theorem 1.3 (i.e., $\left.\Phi_{i} \in \operatorname{Kak}\left(X, Y_{i}\right)\right)$. Let $\Phi: X \rightarrow Y$ be given by

$$
\Phi(x)=\prod_{j=1}^{N_{0}} \Phi_{j}(x) \text { for } x \in X .
$$

Note $\Phi \in \operatorname{Kak}(X, Y)$. Let $\Phi^{\star}$ denote the restriction of $\Phi$ to $Q$ and note $\Phi^{\star} \in \operatorname{Kak}(Q, Y)$. Let $q(y)=$ $\prod_{i=1}^{N} q_{i}(y)$ for $y \in Y$ and note $q \Phi^{\star} \in A d(Q, Q)$. Theorem 1.1 guarantees a $x \in Q$ with $x \in q\left(\Phi^{\star}(x)\right)$. Now let $y \in \Phi^{\star}(x)$ with $x=q(y)$. Note $y \in \Phi(x)$ and $x_{i}=q_{i}(y) \in G_{i}(y)$ for $i \in\{1, \ldots, N\}$. Now from our assumption there exists an $i_{0} \in\left\{1, \ldots, N_{0}\right\}$ with $F_{i_{0}}(x) \neq \emptyset$, i.e., $x \in U_{i_{0}}$ and so $i_{0} \in \Phi_{i_{0}}(x)=F_{i_{0}}(x)$.

Now we will rewrite Theorems 2.13 and 2.14 as maximal element type results.

Theorem 2.15. Let $\left\{X_{i}\right\}_{i=1}^{N},\left\{Y_{i}\right\}_{i=1}^{N_{0}}$ be families of convex sets each in a Hausdorff topological vector space and $\left\{Y_{i}\right\}_{i=1}^{N_{0}}$ is also a family of compact sets. For each $i \in\left\{1, \ldots, N_{0}\right\}$ suppose $F_{i}: X \equiv \prod_{i=1}^{N} X_{i} \rightarrow Y_{i}$ is upper semicontinuous with convex compact values. For each $j \in\{1, \ldots, N\}$ suppose $G_{j}: Y \equiv \prod_{i=1}^{N_{0}} Y_{i} \rightarrow X_{j}$ and $\mathrm{G}_{j} \in \operatorname{DKT}\left(\mathrm{Y}, \mathrm{X}_{\mathrm{j}}\right)$. Now suppose either for each $(x, y) \in \mathrm{X} \times \mathrm{Y}$ there exists $a j \in\left\{1, \ldots, \mathrm{N}_{0}\right\}$ with $\mathrm{y}_{j} \notin \mathrm{F}_{j}(\mathrm{x})$ or for each $(x, y) \in X \times Y$ there exists an $i \in\{1, \ldots, N\}$ with $x_{i} \notin G_{i}(y)$. Then there exists an $x \in X$ and $a$ $i_{0} \in\left\{1, \ldots, \mathrm{N}_{0}\right\}$ with $\mathrm{F}_{\mathrm{i}_{0}}(\mathrm{x})=\emptyset$.

Proof. Suppose the conclusion is false. Then for each $x \in X$ we have $F_{i}(x) \neq \emptyset$ for all $i \in\left\{1, \ldots, N_{0}\right\}$. Thus $F_{i} \in \operatorname{Kak}\left(X, Y_{i}\right)$ for all $i \in\left\{1, \ldots, N_{0}\right\}$. Now Theorem 2.11 guarantees a $x \in X$, a $y \in Y$ with $y_{j} \in F_{j}(x)$ for $j \in\left\{1, \ldots, N_{0}\right\}$ and $x_{i} \in G_{i}(y)$ for $i \in\{1, \ldots, N\}$, a contradiction. 
Theorem 2.16. Let $\left\{X_{i}\right\}_{i=1}^{N},\left\{Y_{i}\right\}_{i=1}^{N_{0}}$ be families of convex sets each in a Hausdorff topological vector space and $\left\{Y_{i}\right\}_{i=1}^{N_{0}}$ is also a family of compact sets. For each $i \in\left\{1, \ldots, N_{0}\right\}$ suppose $F_{i}: X \equiv \prod_{i=1}^{N} X_{i} \rightarrow Y_{i}$ is upper semicontinuous with convex compact values. For each $j \in\{1, \ldots, N\}$ suppose $G_{j}: Y \equiv \prod_{i=1}^{N_{0}} Y_{i} \rightarrow X_{j}$ and $\mathrm{G}_{j} \in \operatorname{DKT}\left(\mathrm{Y}, \mathrm{X}_{j}\right)$. Also assume for each $i \in\left\{1, \ldots, \mathrm{N}_{0}\right\}$ that $\mathrm{U}_{i}=\left\{x \in X: F_{i}(x) \neq \emptyset\right\}$ is open in $\mathrm{X}$. Now suppose either for each $(x, y) \in X \times Y$ we have $y_{j} \notin F_{j}(x)$ for all $j \in\left\{1, \ldots, N_{0}\right\}$ or for each $(x, y) \in X \times Y$ there exists an $i \in\{1, \ldots, N\}$ with $x_{i} \notin G_{i}(y)$. Then there exists an $x \in X$ with $F_{i}(x)=\emptyset$ for all $i \in\left\{1, \ldots, N_{0}\right\}$.

Proof. Suppose the conclusion is false. Then for each $x \in X$ there exists an $i \in\left\{1, \ldots, N_{0}\right\}$ with $F_{i}(x) \neq \emptyset$. Now Theorem 2.14 guarantees a $x \in X$, a $y \in Y$ and a $j_{0} \in\left\{1, \ldots, N_{0}\right\}$ with $y_{j_{0}} \in F_{j_{0}}(x)$ and $x_{i} \in G_{i}(y)$ for $i \in\{1, \ldots, N\}$, a contradiction.

We will now use the discussion after Theorem 2.7 to obtain our final results.

Theorem 2.17. Let $\left\{X_{i}\right\}_{i=1}^{N},\left\{Y_{i}\right\}_{i=1}^{N_{0}}$ be families of convex sets each in a Hausdorff topological vector space, $\left\{Y_{i}\right\}_{i=1}^{N_{0}}$ is also a family of compact sets, and $\mathrm{X} \equiv \prod_{i=1}^{\mathrm{N}} \mathrm{X}_{i}$ is paracompact. For each $i \in\left\{1, \ldots, \mathrm{N}_{0}\right\}$ suppose $\mathrm{H}_{i}: \mathrm{X} \rightarrow \mathrm{Y}_{i}$ and for each $x \in X$ assume there exists a map $A_{i, x}: X \rightarrow Y_{i}$ and an open set $U_{i, x}$ containing $x$ with $H_{i}(z) \subseteq A_{i, x}(z)$ for every $z \in U_{i, x}, A_{i, x}: U_{i, x} \rightarrow Y_{i}$ is upper semicontinuous with convex compact values. For each $j \in\{1, \ldots, N\}$ suppose $G_{j}: Y \equiv \prod_{i=1}^{N_{0}} Y_{i} \rightarrow X_{j}$ and $G_{j} \in \operatorname{DKT}\left(Y, X_{j}\right)$. Also assume either for each $x \in X$ and for each $(w, y) \in X \times Y$ there exists $a j_{0} \in\left\{1, \ldots, N_{0}\right\}$ (which does not depend on $\left.X\right)$ with $y_{j_{0}} \notin A_{j_{0}, x}(w)$ or for each $(x, y) \in X \times Y$ there exists an $i \in\{1, \ldots, N\}$ with $x_{i} \notin G_{i}(y)$. Then there exists an $x \in X$ and $a i_{0} \in\left\{1, \ldots, N_{0}\right\}$ with $\mathrm{H}_{\mathrm{i}_{0}}(\mathrm{x})=\emptyset$.

Proof. Let $i \in\left\{1, \ldots, N_{0}\right\}$. From the discussion after Theorem 2.7 (with $Z=X, W=Y_{i}, H=H_{i}$ and $\left.A_{x}=A_{i, x}\right)$ there exists a map $\Psi_{i}: X \rightarrow Y_{i}$ with $H_{i}(z) \subseteq \Psi_{i}(z)$ for $z \in X$ and $\Psi_{i}: X \rightarrow Y_{i}$ is upper semicontinuous with convex compact values: here $\left\{U_{i, x}\right\}_{x \in X}$ is an open covering of $X$ so there exists a locally finite open covering $\left\{V_{i, x}\right\}_{x \in X}$ of $X$ (recall $X$ is paracompact) with $x \in V_{i, x}$ and $V_{i, x} \subseteq U_{i, x}$ for $x \in X$, and for each $x \in X$,

and $\Psi_{i}: X \rightarrow Y_{i}$ is

$$
Q_{i, x}(z)= \begin{cases}A_{i, x}(z), & z \in V_{i, x} \\ Y_{i,} & z \in X \backslash V_{i, x}\end{cases}
$$

$$
\Psi_{i}(z)=\bigcap_{x \in X} Q_{i, x}(z) \text { for } z \in X .
$$

We now claim for each $x \in X$ and for each $(w, y) \in X \times Y$ there exists a $j_{0} \in\left\{1, \ldots, N_{0}\right\}$ with $y_{j_{0}} \notin \Psi_{j_{0}}(w)$ if in the statement of Theorem 2.17 we have for each $x \in X$ and for each $(w, y) \in X \times Y$ there exists a $j_{0} \in\left\{1, \ldots, N_{0}\right\}$ (which does not depend on $X$ ) with $y_{j_{0}} \notin A_{j_{0}, x}(w)$. To see this fix $(w, y) \in X \times Y$. Now for each $x \in X$ there exists a $j_{0} \in\{1, \ldots, N\}$ (which does not depend on $x$ ) with $y_{j_{0}} \notin A_{j_{0}, x}(w)$. Now since $\left\{V_{j_{0}, x}\right\}_{x \in X}$ is a covering of $X$ there exists an $x^{j_{0}} \in X$ with $w \in V_{j_{0}, x^{j_{0}}}$ so

$$
\Psi_{j_{0}}(w)=\bigcap_{x \in X} Q_{j_{0}, x}(w) \subseteq Q_{j_{0}, x_{0}}(w)=A_{j_{0}, x^{j_{0}}}(w),
$$

and as a result $y_{j_{0}} \notin \Psi_{j_{0}}(w)$. Thus our claim is true. Now apply Theorem 2.15 (with $F_{i}=\Psi_{i}$ ) so there exists an $x \in X$ and $a i_{0} \in\left\{1, \ldots, N_{0}\right\}$ with $\Psi_{i_{0}}(x)=\emptyset$. Now since $H_{i}(z) \subseteq \Psi_{i}(z)$ for $z \in X$, then $H_{i_{0}}(x)=\emptyset$.

Theorem 2.18. Let $\left\{X_{i}\right\}_{i=1}^{N},\left\{Y_{i}\right\}_{i=1}^{N_{0}}$ be families of convex sets each in a Hausdorff topological vector space, $\left\{Y_{i}\right\}_{i=1}^{N_{0}}$ is also a family of compact sets, and $\mathrm{X} \equiv \prod_{i=1}^{\mathrm{N}} \mathrm{X}_{i}$ is paracompact. For each $i \in\left\{1, \ldots, \mathrm{N}_{0}\right\}$ suppose $\mathrm{H}_{i}: \mathrm{X} \rightarrow \mathrm{Y}_{i}$ and for each $x \in X$ assume there exists a map $A_{i, x}: X \rightarrow Y_{i}$ and an open set $U_{i, x}$ containing $x$ with $H_{i}(z) \subseteq$ $A_{i, x}(z)$ for every $z \in U_{i, x}, A_{i, x}: U_{i, x} \rightarrow Y_{i}$ is upper semicontinuous with convex compact values. For each $j \in\{1, \ldots, N\}$ suppose $G_{j}: Y \equiv \prod_{i=1}^{N_{0}} Y_{i} \rightarrow X_{j}$ and $G_{j} \in \operatorname{DKT}\left(Y, X_{j}\right)$. Also assume for each $i \in\left\{1, \ldots, N_{0}\right\}$ that $\mathrm{U}_{i}=\left\{x \in X: \mathrm{F}_{i}(\mathrm{x}) \neq \emptyset\right\}$ is open in $\mathrm{X}$. Finally suppose either for each $\mathrm{x} \in \mathrm{X}$ and for each $(w, y) \in \mathrm{X} \times \mathrm{Y}$ we have $y_{j} \notin A_{j, x}(w)$ for all $j \in\left\{1, \ldots, N_{0}\right\}$ or for each $(x, y) \in X \times Y$ there exists an $i \in\{1, \ldots, N\}$ with $x_{i} \notin G_{i}(y)$. Then there exists an $\mathrm{x} \in \mathrm{X}$ with $\mathrm{H}_{\mathrm{i}}(\mathrm{x})=\emptyset$ for all $\mathrm{i} \in\left\{1, \ldots, \mathrm{N}_{0}\right\}$. 
Proof. Let $i \in\left\{1, \ldots, \mathrm{N}_{0}\right\}$ and let $\mathrm{V}_{i, x}, \mathrm{Q}_{i, x}$ and $\Psi_{i}$ be as in Theorem 2.17. We now claim for each $(w, y) \in$ $X \times Y$ we have $y_{j} \notin \Psi_{j}(w)$ for $j \in\left\{1, \ldots, N_{0}\right\}$ if in the statement of Theorem 2.18 we have for each $x \in X$ and for each $(w, y) \in X \times Y$ we have $y_{j} \notin A_{j, x}(w)$ for all $j \in\left\{1, \ldots, N_{0}\right\}$. To see this fix $(w, y) \in X \times Y$ and fix $i \in\left\{1, \ldots, N_{0}\right\}$. Now for each $x \in X$ we have $y_{j} \notin A_{j, x}(w)$. Since $\left\{V_{i, x}\right\}_{x \in X}$ is a covering of $X$ there exists an $x^{\star} \in X$ with $w \in V_{i, x^{\star}}$ so

$$
\Psi_{i}(w)=\bigcap_{x \in X} Q_{i, x}(w) \subseteq Q_{i, x^{\star}}(w)=A_{i, x^{\star}}(w),
$$

and thus since $y_{i} \notin A_{i, x^{\star}}(w)$ we have $y_{i} \notin \Psi_{i}(w)$, and our claim is true.

Let $i \in\left\{1, \ldots, N_{0}\right\}$ and define a map $\Phi_{i}: X \rightarrow Y_{i}$ by

$$
\Phi_{i}(x)= \begin{cases}\Psi_{i}(x), & x \in U_{i}=\left\{x \in X: H_{i}(x) \neq \emptyset\right\} \\ Y_{i}, & x \in X \backslash U_{i}\end{cases}
$$

Note $\Phi_{i}$ has nonempty convex compact values and $\Phi_{i}: X \rightarrow Y_{i}$ is upper semicontinuous from Theorem 1.3 (i.e., $\left.\Phi_{i} \in \operatorname{Kak}\left(X, Y_{i}\right)\right)$. Let $\Phi: X \rightarrow Y$ be given by

$$
\Phi(x)=\prod_{j=1}^{N} \Phi_{j}(x) \text { for } x \in X .
$$

Note $\Phi \in \operatorname{Kak}(X, Y)$. Let $q_{i}, R_{i}, Q_{i}$ and $Q$ be as in Theorem 2.11 and let $\Phi^{\star}$ denote the restriction of $\Phi$ to $Q$ and note $\Phi^{\star} \in \operatorname{Kak}(Q, Y)$. Now let $q(y)=\prod_{i=1}^{N} q_{i}(y)$ for $y \in Y$ and note $q: Y \rightarrow Q$ is continuous so $q \Phi^{\star} \in \operatorname{Ad}(Q, Q)$. Theorem 1.1 guarantees a $x \in Q$ with $x \in q\left(\Phi^{\star}(x)\right)$. Let $y \in \Phi^{\star}(x)$ with $x=q(y)$, i.e., $y \in \Phi(x)$ with $x_{i}=q_{i}(y)$ for $i \in\left\{1, \ldots, N_{0}\right\}$. For $i \in\left\{1, \ldots, N_{0}\right\}$ we have $x_{i}=q_{i}(y) \in G_{i}(y)$ which is a contradiction if we assume in the statement of Theorem 2.18 that for each $(x, y) \in X \times Y$ there exists an $i \in\{1, \ldots, N\}$ with $x_{i} \notin G_{i}(y)$. Suppose there exists an $i_{0} \in\left\{1, \ldots, N_{0}\right\}$ with $H_{i_{0}}(x) \neq \emptyset$. Then $x \in U_{i_{0}}$ so we have $y_{i_{0}} \in \Phi_{i_{0}}(x)=\Psi_{i_{0}}(x)$, which is a contradiction if we assume in the statement of Theorem 2.18 that for each $z \in X$ and for each $(w, y) \in X \times Y$ we have $y_{j} \notin A_{j, z}(w)$ for all $j \in\left\{1, \ldots, N_{0}\right\}$. As a result $\mathrm{H}_{i}(\mathrm{x})=\emptyset$ for all $i \in\left\{1, \ldots, \mathrm{N}_{0}\right\}$.

\section{References}

[1] R. P. Agarwal, D. O'Regan, S. Park, Fixed point theory for multimaps in extension type spaces, J. Korean Math. Soc., 39 (2002), 579-591. 1.1

[2] C. D. Aliprantis, K. C. Border, Infinite-Dimensional Analysis, Studies in Economic Theory, Springer-Verlag, Berlin, (1994). 1, 2

[3] H. Ben-El-Mechaiekh, P. Deguire, A. Granas, Points fixes et coincidences pour les fonctions multivoques (Applications de type $\Phi$ and $\left.\Phi^{\star}\right)$ II, C. R. Acad. Sci. Paris Sér. I Math., 295 (1982), 381-384. 1, 2

[4] X. P. Ding, W. K. Kim, K. K. Tan, A selection theorem and its applications, Bulletin Australian Math. Soc., 46 (1992), 205-212. 1, 1, 2.8, 2

[5] J. Dugundji, Topology, Allyn and Bacon, Boston, (1966). 2

[6] R. Engelking, General Topology, PWN-Polish Scientific Publishers, Warszawa, (1977). 2

[7] L. Gorniewicz, Topological fixed point theory of multivalued mappings, Kluwer Academic Publishers, Dordrecht, (1999). 1

[8] D. O'Regan, Fixed point theory on extension type spaces on topological spaces, Fixed Point Theory and Applications, 1 (2004), 13-20. 1.1

[9] D. O'Regan, Deterministic and random fixed points for maps on extension type spaces, Appl. Anal., 97 (2018), $1960-1966$. 1.2

[10] D. O'Regan, Collectively coincidence type results and applications, Appl. Anal., 2021 (2021), 12 pages. 1, 2

[11] D. O'Regan, A note on collectively fixed and coincidence points, to appear. 1, 1

[12] K.-K. Tan, X.-Z. Yuan, Maximal elements and equilibria for U-majorised preferences, Bull. Austral. Math. Soc., 49 (1994), 47-54. 1, 1, 2 